\title{
Acquired and Natural Immunity to Gonococcal Infection
}

\section{in Chimpanzees}

\author{
Stephen J. Kraus, W. Jerry Brown, and Robert J. Arko \\ From the Center for Disease Control, Public Health Service, U. S. Department \\ of Health, Education, and Welfare, Atlanta, Georgia 30.333
}

\begin{abstract}
A B S T R A C T Despite the fact that gonorrhea is our most common reportable infectious disease, little is known about natural and acquired resistance to Neisseria gonorrhoeae. With the chimpanzee model, which mimics human gonococcal infection in signs, symptoms, and host response, a natural resistance to gonococcal challenge was found. One aspect of this natural resistance became evident when the cervix and oral pharynx resisted more gonococci than the urethra. Natural resistance was also shown when environmental factors were found to influence resistance to gonococcal pharyngitis. In addition to natural resistance a postinfectionacquired immunity to the gonococcus was demonstrated. Following gonococcal pharyngitis, this anatomical location successfully resisted more gonococci than were initially resisted. Similarly, more gonococci were successfully resisted in rechallenging the urethra. These findings are related to the clinic situation and suggest possible new approaches to gonorrhea control.
\end{abstract}

\section{INTRODUCTION}

It is not unusual for a patient to contract gonococcal urethritis many times. The development of clinically significant immunity to reinfection after gonorrhea is questioned by clinicians when treating the same patients who return with the same problem a week or a month after treatment for gonorrhea. The paradox of gonorrhea has recently been used to describe those patients who become reinfected despite significant increases of antigonococcal systemic antibody, local secretory $\operatorname{IgA}$, and cell-mediated immunity (1).

Contrasting with the repetitiousness of gonorrhea in some individuals is the fact that only one in five men

A preliminary report was presented at the 13th Interscience Conference on Antimicrobial Agents and Chemotherapy, Washington, D. C., September 1973.

Received for publication $25 \mathrm{July} 1974$ and in revised form 17 February 1975. who experience a single sexual contact with an infected female develop gonorrhea (2). The mechanism (or mechanisms) for this resistance is (or are) not understood, but natural or acquired immunity could possibly account for a portion of the $80 \%$ who escape infection.

Human volunteer experiments might be one avenue for answering the question of natural and acquired immunity in gonococcal resistance. Experimentally induced gonococcal urethritis had been accomplished as early as 1767 (3), but more recent attempts to establish a reproducible infective technique were abandoned after an exhaustive number of experiments (4). Somewhat later a reproducible technique was described (5), but the infections were terminated after 5 days (6), because of potential problems involving the psychological and general health of the volunteers. ${ }^{1}$ Such a short duration of infection was insufficient time for the host to develop immunity capable of terminating the infection, and, indeed, the time required for spontaneous termination of gonorrhea may exceed several months (7).

An animal model has recently been described which mimics uncomplicated gonococcal infection in man with respect to incubation period, certain clinical manifestations, sexual transmission, local cellular response in the exudate, in vitro culture characteristics of the infection material, and systemic immune response (8-10). This animal model has now been used to answer the basic question: does immunity to reinfection develop in the course of a gonococcal infection? If immunity does ensue, related questions include the following: is it absolute or relative, and is it related to the dynamics of the initial infection, such as infective dose and duration of the prior infection (7). During these experiments other information was obtained, such as that concerning the minimal infective inoculum of gonococci; natural immunity to Neisseria gonorrhoeae: gonococcal viru-

${ }^{1}$ Kellogg, D. S. Personal communication. 
lence determinants other than colony type $(5,11,12)$ and pili $(13,14)$; infectivity of colony type 5 (15); and the dynamics of gonorrhea as influenced by its anatomical location (urethra, cervix, or pharynx).

\section{METHODS}

Chimpanzees. 16 adult chimpanzees participated in either all or a portion of the experiments. Not all chimpanzees participated in all experiments because of logistical and technical problems in handling adult chimpanzees. Three of the animals were female (nos. 12,13, and 16). The animals were housed according to their compatibility, and usually chimpanzees of the same sex were caged together. If the sexes were mixed, sexual activity was noted, and this was documented only with animal 2 and a female not included in this study. Three chimpanzees (nos. 14, 15, and 16) were housed in indoor facilities at the Center for Disease Control (CDC chimpanzees) ${ }^{2}$ and the others were kept in outdoor facilities at Chamblee, Ga. (CHB chimpanzees). Six of the animals (nos. 4, 6, 7, 10, 11, and 12) had been infected with $N$. gonorrhoeae in the urethra or cervix $2 \mathrm{yr}$ previously. The remainder had had no prior gonococcal infection.

Gonococcal inocula. Two clinical isolates of $N$. gonorrhoeae ( $\mathrm{C}$ and $\mathrm{M}$ ) were obtained from men with gonococcal urethritis. These were isolated on selective media (16) and identified as $N$. gonorrhoeae on the basis of Gram stain, colonial morphology, oxidase reaction (utilizing a $1 \%$ solution of $N, N$-dimethyl- $\rho$-phenylenediamine monohydrochloride), and fermentation of dextose but not maltose, sucrose, lactose, fructose, or mannitol (fermentation medium consisted of $1 \%$ test carbohydrate in cystine tryptic agar medium). After two in vitro passes on GCB medium (GC base medium plus Isovitalex) ${ }^{3}$ to ensure purity, the isolates were frozen at $-90^{\circ} \mathrm{C}$ in phosphate-buffered saline, $\mathrm{pH} 7.2$, containing $10 \%$ glycerol. Multiple vials were frozen so that all challenges with $C$ and $M$ isolates were performed with gonococci that had not been passed in vitro more than three times.

Gonocccal isolate $T$ was a laboratory strain passed in vitro on GCB medium more than 500 times. These passes were selectively done to maintain colony type 1 (11). Isolate $K_{2} 43562$ was supplied by A. Reyn (Neisseria Department, Staten Seruminstitute, Copenhagen, Denmark) and was colony type 5 (15).

Gonococci for chimpanzee inoculation were grown for $18 \mathrm{~h}$ in GCB medium. C, M, and T inocula consisted of greater than $95 \%$ colony type 1 , and $K_{2} 43562$ was greater than $95 \%$ colony type 5 . These gonococci were removed from the agar surface with sterile cotton-tipped applicators and suspended in Trypticase soy broth to an optical density of 0.3 at $530 \mathrm{~nm}$ (approximately $10^{\circ}$ colony forming units [CFU]/ $\mathrm{ml}$. An inoculum above $10^{\circ} \mathrm{CFU}$ was obtained by adding gonococci from several Petri dishes to the soy broth. Log dilutions by a factor of 10 of the gonococci were made in

${ }^{2}$ Abbreviations used in this paper: C, M, T, $\mathrm{K}_{2} 43562$, clinical isolates; CDC, Center for Disease Control; CFU, colony-forming units; $\mathrm{CHB}$, chimpanzees kept at Chamblee, Ga.; MCD, maximal challenge dose; MID, minimal infective dose; MRD, maximal resistance dose; VCNT, vancomycin, colistin, nystatin, and trimethoprim.

${ }^{3}$ Use of trade names is for identification only and does not constitute endorsement by the Public Health Service or by the U. S. Department of Health, Education, and Welfare. the same broth, and pour plates were made from the higher dilutions. CFU were counted on that plate with the greatest number of discrete colonies. The sequence of gonococcal isolates inoculated into the chimpanzees was $\mathrm{C}, \mathrm{T}$, and $\mathrm{M}$.

Chimpanzee inoculation. Urethral inoculations were similar to that previously described (8). $1 \mathrm{ml}$ of gonococcal suspension was introduced into the urethra by means of a plastic catheter. The number of gonococci inoculated into the first chimpanzees studied in the experiments varied until an understanding of the magnitude of minimal infective dose (MID) was ascertained. In these early experiments the dose used to infect was therefore not necessarily the MID, since smaller doses might also have infected. Subsequent initial inocula in other chimps were started at $100 \mathrm{CFU}$, and then challenge doses were increased by a factor of 10 until either infection occurred or high challenge doses were successfully resisted. The latter constituted the maximal challenge dose (MCD), and it did not necessarily reflect the maximal resistance dose (MRD), since greater numbers of gonococci may have been resisted. If infection occurred, the MIDs were calculated as well as the MRDs which, according to the protocol, were usually a factor of 10 less than the MIDs.

Pharyngeal inoculation was done with the animal in a supine position. The tongue was depressed and $1 \mathrm{ml}$ of gonococcal suspension was placed over the tonsils and into the oral pharynx. The initial inoculum contained $100 \mathrm{CFU}$, and subsequent inocula were progressively increased in a manner identical to that employed in the urethral challenges.

Cervical inoculation was performed with the chimpanzee in the lithotomy position, and the cervix was observed with the aide of a vaginal speculum. $1 \mathrm{ml}$ of gonococcal suspension was placed onto the cervix with a pipette. The initial inoculum was $100 \mathrm{CFU}$, and this was increased by a factor of 10 until either infection occurred or a high challenge dose was resisted.

Detection of gonococcal infection. Specimens from the animals were cultured within a week after inoculation and weekly thereafter if a gonococcal infection was induced. Two consecutive negative cultures were obtained to document failure to infect or termination of infection.

Urethral specimens were obtained by inserting a bacterial loop $6 \mathrm{~cm}$ into the urethra. Cervical specimens were collected on a cotton-tipped applicator inserted into the cervical os after the latter was observed with a vaginal speculum. The rectal area was sampled by introducing a sterile cottontipped applicator $3 \mathrm{~cm}$ into the rectum. Oral pharynx specimens were obtained with a cotton-tipped applicator touched to both tonsils and the posterior aspect of the oral pharynx. All specimens were plated onto selective medium (16) and vancomycin, colistin, nystatin, and trimethoprim (VCNT) medium (selective medium without hemoglobin and containing $5 \mu \mathrm{g} / \mathrm{ml}$ of trimethoprim). The VCNT medium allowed colony typing of the primary isolate (17); it also suppressed an occasional Proteus which overgrew the surface of the standard selective medium (18).

All cultures were incubated at $36.5^{\circ} \mathrm{C}$ in a candle extinction jar and examined for growth at 24 and $48 \mathrm{~h}$. Oxidase-positive colonies were further studied by Gram stain and sugar fermentation.

Rechallenge inoculations. Within 1 wk after termination of the initial infection with $M$ isolate, the animals were rechallenged with $M$ isolate starting with $10^{3}$ CFU and proceeding as in the primary challenge until reinfection occurred or a high challenge dose failed to reinfect. Those animals reinfected with $M$ isolate were cultured weekly until spontaneous termination of the infection. 
TABLE I

Resistance, Ineffective Inoculum, and Duration of Gonococcal Urethritis in the Initial Infection and in the Rechallenge Experiment

\begin{tabular}{|c|c|c|c|c|c|c|c|c|c|}
\hline \multirow[b]{2}{*}{ Chimp } & \multicolumn{4}{|c|}{ Initial infection } & \multicolumn{4}{|c|}{ Rechallenge } & \multirow[b]{2}{*}{$\begin{array}{c}\text { Prior* } \\
\text { gonorrhea }\end{array}$} \\
\hline & $\begin{array}{l}\text { Infection } \\
\text { inoculum }\end{array}$ & MID & MRD & $\begin{array}{l}\text { Infection } \\
\text { duration }\end{array}$ & MID & MRD & MCD & $\begin{array}{l}\text { Infection } \\
\text { duration }\end{array}$ & \\
\hline & $C F U$ & \multicolumn{2}{|c|}{$C F U$} & days & & $C F U$ & & days & \\
\hline 1 & $4.0 \times 10^{4}$ & $4.0 \times 10^{4}$ & $1.4 \times 10^{3}$ & 40 & $7.8 \times 10^{6}$ & $6.0 \times 10^{5}$ & & 14 & 0 \\
\hline 2 & $1.0 \times 10^{9}$ & ND & ND & $70 \ddagger$ & & & $1.4 \times 10^{11}$ & $N R$ & 0 \\
\hline 3 & $6.9 \times 10^{9}$ & ND & ND & 21 & $5.1 \times 10^{8}$ & $6.0 \times 10^{7}$ & & 36 & 0 \\
\hline 4 & $3.5 \times 10^{3}$ & $3.5 \times 10^{3}$ & $3.5 \times 10^{2}$ & 50 & $7.0 \times 10^{6}$ & $4.7 \times 10^{5}$ & & 19 & + \\
\hline 5 & $6.9 \times 10^{6}$ & ND & ND & 21 & $5.1 \times 10^{8}$ & $6.0 \times 10^{7}$ & & 14 & 0 \\
\hline 6 & $7.8 \times 10^{3}$ & $7.8 \times 10^{3}$ & $7.8 \times 10^{2}$ & 21 & $1.1 \times 10^{7}$ & $1.0 \times 10^{6}$ & & 5 & + \\
\hline 7 & $4.0 \times 10^{4}$ & $4.0 \times 10^{4}$ & $1.4 \times 10^{3}$ & 27 & $7.8 \times 10^{6}$ & $6.0 \times 10^{5}$ & & 25 & + \\
\hline 8 & $1.8 \times 10^{8}$ & ND & ND & 41 & & & $1.4 \times 10^{11}$ & $\mathrm{NR}$ & 0 \\
\hline 9 & $3.4 \times 10^{5}$ & ND & ND & 27 & $4.6 \times 10^{6}$ & $5.1 \times 10^{5}$ & & 6 & 0 \\
\hline 10 & $7.0 \times 10^{5}$ & $7.0 \times 10^{5}$ & $1.2 \times 10^{4}$ & 23 & & & $6.0 \times 10^{10}$ & NR & + \\
\hline 11 & $3.4 \times 10^{5}$ & ND & ND & 20 & & & $5.2 \times 10^{9}$ & $N R$ & + \\
\hline
\end{tabular}

ND, not determined; NR, not reinfected.

* Gonococcal urethritis with spontaneous cure $2 \mathrm{yr}$ earlier.

$\ddagger$ Possible reinfection from cagemate.

\section{RESULTS}

11 male chimpanzees participated in various aspects of the urethral challenges with $N$. gonorrhoeae. The first two gonococcal isolates inoculated into the urethras (isolates $\mathrm{C}$ and $\mathrm{T}$ ) failed to induce urethritis. Five chimpanzees (nos. 1, 3, 5, 8, and 9) were challenged with $C$ isolate, and the MCD varied from $10^{8}$ to $10^{10}$ CFU. Three chimpanzees (nos. 3, 5, and 8) were then challenged with $\mathrm{T}$ isolate, and the MCDs ranged from $10^{8}$ to $10^{9} \mathrm{CFU}$. The colony types of these two inocula were greater than $95 \%$ type 1, and they were piliated, as shown by negative staining and transmission electron microscopy $(13,14)$.

In contrast to the failure to induce urethritis with isolates $\mathrm{C}$ and $\mathrm{T}$, all 11 male chimpanzees challenged

\section{TABLE II}

Inoculation Size and the Duration of Urethritis in First Infection with $M$ Isolate

\begin{tabular}{cc}
\hline Inoculum & $\begin{array}{c}\text { Infection } \\
\text { duration }\end{array}$ \\
\hline$C F U$ & days \\
$6.9 \times 10^{9}$ & 21 \\
$1.8 \times 10^{8}$ & 41 \\
$6.9 \times 10^{6}$ & 21 \\
$7.0 \times 10^{5}$ & 23 \\
$3.4 \times 10^{5}$ & 27 \\
$3.4 \times 10^{5}$ & 20 \\
$4.0 \times 10^{4}$ & 27 \\
$4.0 \times 10^{4}$ & 40 \\
$7.8 \times 10^{3}$ & 21 \\
$3.5 \times 10^{3}$ & 50
\end{tabular}

with $\mathrm{M}$ isolate were infected. The data on these urethral challenges is presented in Table I. MIDs and MRDs were determined in five of the chimpanzees: MIDs varied from $3.5 \times 10^{3}$ to $7.0 \times 10^{5} \mathrm{CFU}$, and the MRDs from $3.5 \times 10^{2}$ to $1.2 \times 10^{4} \mathrm{CFU}$. The geometric mean MRD for these five animals was $1.4 \times 10^{3}$ and the geometric mean MID was $3.1 \times 10^{4}$. The dose used to infect the urethras of the other six animals may or may not be MIDs, since the first inoculum introduced into these chimpanzees produced urethritis. The duration of the infection with $\mathrm{M}$ isolate in 10 chimpanzees varied from 20 to 50 days, with a mean of 29 days. Chimpanzee 2 remained infected for 70 days, but he was caged with a female whom he infected, and the 70-day infection may have been secondary to repeated infection from the cagemate. Table II shows that there was no

TABLE III

$M R D$ of $M$ Isolate in CFU Inoculated into the Urethra of 11 Chimpanzees

\begin{tabular}{cccc}
\hline Chimp & $\begin{array}{c}\text { Initial infection } \\
\text { MRD }\end{array}$ & $\begin{array}{c}\text { Rechallenge } \\
\text { MRD }\end{array}$ & $\begin{array}{c}\text { Sign of } \\
\text { difference }\end{array}$ \\
\hline 1 & $1.4 \times 10^{3}$ & $6.0 \times 10^{5}$ & + \\
2 & $<1.0 \times 10^{9}$ & $\geq 1.4 \times 10^{11}$ & + \\
3 & $<6.9 \times 10^{9}$ & $6.0 \times 10^{7}$ & $?$ \\
4 & $3.5 \times 10^{2}$ & $4.7 \times 10^{5}$ & + \\
5 & $<6.9 \times 10^{6}$ & $6.0 \times 10^{7}$ & + \\
6 & $7.8 \times 10^{2}$ & $1.0 \times 10^{6}$ & + \\
7 & $1.4 \times 10^{3}$ & $6.0 \times 10^{5}$ & + \\
8 & $<1.8 \times 10^{8}$ & $\geq 1.4 \times 10^{11}$ & + \\
9 & $<3.4 \times 10^{5}$ & $5.1 \times 10^{5}$ & + \\
10 & $1.2 \times 10^{4}$ & $\geq 6.0 \times 10^{10}$ & + \\
11 & $<3.4 \times 10^{5}$ & $\geq 5.2 \times 10^{9}$ & + \\
Geometric mean: Max $=3.4 \times 10^{5}$ & Min $=8.6 \times 10^{7}$ & \\
\hline
\end{tabular}


TABLE IV

Duration of the Initial Urethritis and the Degree of Resistance to Rechallenge with the Same Isolate

\begin{tabular}{cc}
\hline $\begin{array}{c}\text { Infection } \\
\text { duration }\end{array}$ & Resistance \\
\hline days & $C F U$ \\
70 & $1.4 \times 10^{11}$ \\
50 & $4.7 \times 10^{5}$ \\
41 & $1.4 \times 10^{11}$ \\
40 & $6.0 \times 10^{5}$ \\
27 & $6.0 \times 10^{5}$ \\
27 & $5.1 \times 10^{5}$ \\
23 & $6.0 \times 10^{10}$ \\
21 & $1.0 \times 10^{6}$ \\
21 & $6.0 \times 10^{7}$ \\
21 & $6.0 \times 10^{7}$ \\
20 & $5.2 \times 10^{9}$ \\
\hline
\end{tabular}

apparent relationship between the inoculum size used to induce urethritis and the duration of the initial infection with $\mathrm{M}$ isolate.

Upon rechallenge with $M$ isolate, 4 of the 11 chimpanzees failed to be infected with MCDs ranging from $5.2 \times 10^{\circ}$ to $1.4 \times 10^{11} \mathrm{CFU}$ (geometric mean $=5.0 \times$ $\left.10^{10}\right)$. Among the seven chimps reinfected, the MRDs ranged from $4.7 \times 10^{5}$ to $6.0 \times 10^{7} \mathrm{CFU}$ (geometric mean $\left.=2.3 \times 10^{\circ}\right)$, and MIDs ranged from $4.6 \times 10^{\circ}$ to $5.1 \times 10^{8} \mathrm{CFU}$ (geometric mean $=2.5 \times 10^{7}$ ). The geometric mean of the maximal number of gonococci resisted (MRD or MCD) by all 11 chimps was $8.6 \times$ $10^{7} \mathrm{CFU}$. The same value for the five chimps in which initial infection MRDs were determined was $6.3 \times 10^{\circ}$. In Table III MRDs from the initial infection are presented with the corresponding data from the rechallenge experiments. Actual initial infection MRD values were available for 5 of the 11 chimps. For use in Table III, the initial infection MRD for the other six animals was taken as a number less than the infective inoculum. Actual rechallenge MRDs were available for seven chimps; for the other four, the MRD was taken as a number equal to or greater than the MCD. The Sign test applied to the data in Table III provides strong evidence $(P<0.001)$ that the rechallenge MRD was substantially greater than the initial infection MRD. Chimpanzee 2 was included in the rechallenge experiments after he and his cagemate spontaneously terminated their genital infection.

The duration of the second urethral infection with $\mathrm{M}$ isolate ranged from 5 to 36 days with a mean of 17 days. This mean duration was shorter than the corresponding mean duration of the initial infection (29 days). The duration of the second urethritis was shorter than in the initial urethritis for six of the seven animals reinfected, but this was not statistically significant $(P=0.06$ Sign test).

The relationship between the duration of the first urethral infection with $\mathrm{M}$ isolate and the degree of resistance to reinfection upon rechallenge with the same isolate is outlined in Table IV. The latter is represented by the MRD in those chimpanzees reinfected or the MCD in those that resisted reinfection. As shown in the table, the duration of the first infection showed no apparent relationship to the degree of resistance to rechallenge.

5 of these 11 male chimpanzees had experienced gonococcal urethritis $2 \mathrm{yr}$ previously. MRDs in the first infection with $M$ isolate were determined in four of these animals, and they ranged from $3.5 \times 10^{2}$ to $1.2 \times 10^{4} \mathrm{CFU}$ with a geometric mean of $1.5 \times 10^{3}$ CFU. Of the six other chimpanzees infected with $\mathrm{M}$ isolate (those without prior gonorrhea), an MRD was determined in only one animal, and this was $1.4 \times 10^{3}$ CFU. The duration of the first infection with $M$ isolate in the five chimpanzees infected previously ranged from 20 to 50 days, with a mean of 28 days. The duration of the first infection with $\mathrm{M}$ isolate in the other six chimpanzees was 21 to 41 days, with a mean of 30 days.

The cervices of two female animals were inoculated with $M$ isolate. One resisted infection (MCD of $1.0 \times$ $10^{12} \mathrm{CFU}$ ), and the other was infected with $5.1 \times 10^{8}$ CFU after resisting $5.0 \times 10^{7}$ CFU (Table V). The geometric mean of the maximal number of gonococci resisted by these two animals in the cervical area was $2.2 \times 10^{\circ} \mathrm{CFU}$.

All 16 chimpanzees participated in various aspects of the pharyngeal challenges with $N$. gonorrhoeae.

TABLE V

Resistance, Infective Inoculum, and Duration of Gonococcal Cervicitis in the Chimpanzee

\begin{tabular}{ccccccc}
\hline Chimp & Infection & MID & MRD & MCD & $\begin{array}{c}\text { Infection } \\
\text { duration }\end{array}$ & $\begin{array}{c}\text { Prior } \\
\text { gonorrhea }\end{array}$ \\
\hline & & & & & days & \\
12 & + & $5.1 \times 10^{8}$ & $5.0 \times 10^{7}$ & & 15 & + \\
13 & 0 & & & $1.0 \times 10^{11}$ & & 0 \\
\hline
\end{tabular}


TABLE VI

Resistance, Infective Inoculum, and Duration of Gonococcal Pharyngitis in the Initial Infection and the Rechallenge Experiment

\begin{tabular}{|c|c|c|c|c|c|c|c|c|c|c|}
\hline \multirow[b]{3}{*}{ Chimp } & \multirow{3}{*}{$\begin{array}{c}\text { Housing } \\
\text { locale }\end{array}$} & \multicolumn{5}{|c|}{ Initial infection } & \multirow{2}{*}{\multicolumn{2}{|c|}{ Rechallenge }} & \multirow{2}{*}{\multicolumn{2}{|c|}{ Pharyngeal Neisseria* }} \\
\hline & & & & & & & & & & \\
\hline & & Infection & MID & MRD & MCD & duration & Infection & $\mathrm{MCD}$ & N. meningitidis & N. lactamica \\
\hline & & & & $C F U$ & & days & & $C F U$ & & \\
\hline 1 & $\mathrm{CHB}$ & + & $7.0 \times 10^{7}$ & $4.7 \times 10^{6}$ & & 5 & & & + & + \\
\hline 2 & $\mathrm{CHB}$ & 0 & & & $1.0 \times 10^{10}$ & & & & + & + \\
\hline 3 & $\mathrm{CHB}$ & 0 & & & $5.1 \times 10^{8}$ & & & & + & 0 \\
\hline 4 & $\mathrm{CHB}$ & + & $1.3 \times 10^{9}$ & $6.9 \times 10^{8}$ & & 10 & & & + & + \\
\hline 5 & $\mathrm{CHB}$ & 0 & & & $5.1 \times 10^{8}$ & & & & + & 0 \\
\hline 6 & $\mathrm{CHB}$ & + & $1.0 \times 10^{6}$ & $6.0 \times 10^{5}$ & & 6 & & & + & + \\
\hline 7 & $\mathrm{CHB}$ & + & $4.0 \times 10^{4}$ & $1.4 \times 10^{3}$ & & 12 & & & + & 0 \\
\hline 8 & $\mathrm{CHB}$ & 0 & & & $1.0 \times 10^{10}$ & & & & + & + \\
\hline 9 & $\mathrm{CHB}$ & $\mathbf{0}$ & & & $1.0 \times 10^{10}$ & & & & + & + \\
\hline 10 & $\mathrm{CHB}$ & 0 & & & $7.3 \times 10^{10}$ & & & & + & + \\
\hline 11 & $\mathrm{CHB}$ & 0 & & & $1.0 \times 10^{10}$ & & & & 0 & + \\
\hline 12 & СНB & 0 & & & $1.0 \times 10^{11}$ & & & & + & 0 \\
\hline 13 & $\mathrm{CHB}$ & 0 & & & $1.0 \times 10^{11}$ & & & & + & 0 \\
\hline 14 & $\mathrm{CDC}$ & + & $9.8 \times 10^{5}$ & $5.0 \times 10^{4}$ & & 40 & 0 & $1.8 \times 10^{10}$ & 0 & 0 \\
\hline 15 & $\mathrm{CDC}$ & + & $5.0 \times 10^{4}$ & $7.8 \times 10^{3}$ & & 22 & 0 & $1.8 \times 10^{10}$ & 0 & 0 \\
\hline 16 & $\mathrm{CDC}$ & + & $5.0 \times 10^{4}$ & $7.8 \times 10^{3}$ & & 49 & 0 & $1.8 \times 10^{10}$ & 0 & 0 \\
\hline
\end{tabular}

* Isolation of these other Neisseria on one or more occasions.

Chimpanzees had not been previously exposed to gonococci in this anatomical location. Data on pharyngeal challenge, infection, and rechallenge are presented in Table VI. All three chimpanzees challenged at the CDC facilities developed gonococcal pharyngitis, whereas only 4 of 13 chimpanzees housed at the $\mathrm{CHB}$ facilities were infected. The duration of the infection in the $\mathrm{CHB}$ animals ranged from 5 to 12 days, with a mean of 8.2 days, and the range in the CDC animals was from 22 to 49 days, with a mean of 37 days. The MIDs for the $\mathrm{CHB}$ chimpanzees varied from $4.0 \times 10^{4}$ to $1.3 \times 10^{\circ}$, with a geometric mean of $1.4 \times 10^{7}$, and those for the CDC chimpanzees varied from $5.0 \times 10^{4}$ to $9.8 \times 10^{5}$, with a geometric mean of $1.4 \times 10^{5}$. The MRDs for the $\mathrm{CDC}$ animals varied from $7.8 \times 10^{3}$ to $5.0 \times 10^{4}$ with a geometric mean of $1.5 \times 10^{4}$, and the maximal number of gonococci resisted ( $\mathrm{CFU}$ ) by the $\mathrm{CHB}$ animals (MRD or MCD) varied from $1.4 \times 10^{3}$ to $1.0 \times 10^{11}$, with a geometric mean of $6.7 \times 10^{8}$. Statistically significant differences between the pharyngeal infections of the $\mathrm{CDC}$ and $\mathrm{CHB}$ chimpanzees were therefore observed with respect to gonococci successfully resisted $(P$ $<0.01$ Rank test) and duration of infection $(P<0.05$ Rank test).

Only those chimpanzees housed at CDC were available for rechallenge, and this was done with $\mathrm{M}$ isolate. Reinfections did not occur in these three animals, and the $\mathrm{MCD}$ in all three instances was $1.8 \times 10^{10} \mathrm{CFU}$. The MCDs in the pharyngeal rechallenges were all greater than the MRDs from the initial pharyngeal infection.
Neisseria meningitidis (group B) and Neisseria lactamica were frequently isolated from the pharyngeal area of the $\mathrm{CHB}$ animals (Table VI). These Neisseria were never isolated from the CDC animals. Gonococci were never isolated simultaneously with meningococci, but on one occasion they were isolated simultaneously with $N$. lactamica.

The two chimpanzees challenged with $\mathrm{K}_{2} 43562$ resisted infection in the urethra and pharynx with MCDs of $10^{10} \mathrm{CFU}$ to both sites.

The recoverability of $N$. gonorrhoeae on conventional selective medium and on VCNT was comparable except in those rare instances where Proteus overgrowth excluded detection of gonococci on the conventional medium. No rectal cultures were positive for $N$. gonorrhoeae. The colony types of the primary isolates from the throat, cervix, and urethra were a combination of types 1 and 2 , with type 1 predominating in all instances.

\section{DISCUSSION}

This study demonstrates that an acquired immunity to reinfection very likely develops during an infection with $N$. gonorrhoeae. The apparent immunity was demonstrated in both gonococcal urethritis and gonococcal pharyngitis. The degree of immunity was best reflected in the comparison of numbers of gonococci successfully resisted (MRD) before the initial infection to the number resisted before reinfection or the maximal number tested (MCD) in those chimpanzees that successfully resisted reinfection. Initial infection ureth- 
ral MRDs were determined for five chimpanzees, and the geometric mean was $1.4 \times 10^{3} \mathrm{CFU}$. Resistance upon rechallenge was $6.3 \times 10^{6}$ for these 5 animals and $8.6 \times 10^{7}$ for all 11 animals. The degree of resistance on rechallenge was unrelated to the duration of the initial infection, and the latter was unrelated to the size of the inoculum employed to induce the initial infection. With respect to the chimpanzees with gonococcal pharyngitis that were rechallenged, the number of gonococci resisted (geometric mean $=1.8 \times 10^{10}$ ) was 1 million times as many as the number resisted in the initial challenge (geometric mean $=1.4 \times 10^{4}$ ).

This study also demonstrates that besides the apparent acquired immunity due to prior infection, there is also a natural resistance to initial infection. Evidence for this includes differences in numbers of gonococci successfullly resisted in the different anatomical sites commonly involved in gonococcal infections: the urethra, cervix, and oral pharynx. The numbers (geometric mean values) successfully resisted in the $\mathrm{CHB}$ chimpanzees were urethra, $1.4 \times 10^{3} \mathrm{CFU}$; cervix, 2.2 $\times 10^{9} \mathrm{CFU}$; and oral pharynx, $1.6 \times 10^{8} \mathrm{CFU}$. Thus, the cervix and pharynx were resistant to approximately 100 thousand to 1 million more gonococci than the urethra. Other evidence for a natural immunity to gonorrhea was the difference in the resistance to gonococcal pharyngitis in relation to the geographic location of the chimpanzees i.e., differences between the $\mathrm{CHB}$ and the $\mathrm{CDC}$ animals. The $\mathrm{CHB}$ chimpanzees resisted 50 thousand times as many gonococci placed into the oral pharynx as the $\mathrm{CDC}$ animals $\left(6.7 \times 10^{8}\right.$ vs. $1.4 \times 10^{4}$ $\mathrm{CFU})$. Anatomical and environmental factors can therefore influence the degree of resistance to infection with $N$. gonorrhoeae. The mechanism (mechanisms) for this natural resistance is (are) not understood, and our data do not give answers to the questions posed. $N$. meningitidis and $N$. lactamica, however, were frequently isolated from the oral pharynx of the $\mathrm{CHB}$ chimpanzees, but they were never found in the CDC chimpanzees. N.meningitidis and N.lactamica were occasionally simultaneously present in the oral pharynx, but both were absent when the gonococcus was present in the oral pharynx. Bacterial interference in which gonococci are inhibited by meningococci has recently been described and suggested as a possible mechanism for a patient's resistance to gonococcal infection (19). The $N$. meningitidis from the throats of the $\mathrm{CHB}$ chimpanzees did inhibit all three $(\mathrm{C}, \mathrm{T}$, and M) gonococcal isolates.

Although our data demonstrate that a high degree of acquired immunity does develop in gonococcal urethritis and pharyngitis, it does not explain the clinical observation of two or more gonorrheal episodes in the same patient within a 1 - or 2-mo interval. Chimpanzees may differ from man in the handling of the gonococcus in some unknown manner, but prior investigations have been shown that chimpanzee gonococcal urethritis mimics the disease in man with respect to clinical signs, incubation period, sexual transmission, Gram stain of the urethral exudate, cellular response in the exudate, in vitro culture characteristics of the infection material, and systemic immune response (8-10). Despite these similarities at least three major differences may exist between our experimental chimpanzee system and the natural infection in man.

In naturally acquired gonococcal urethritis the incubation period is typically 3 days (20), and patients usually present themselves for treatment within 2 or 3 days after the onset of symptoms (21). Treatment therefore arrests the infection within a week's duration, whereas the initial infection in our experimental urethritis ranged from 20 to 50 days, with a mean of 29.1 days, and the infection was allowed to terminate spontaneously. Although our data do not elucidate the mechanism of acquired immunity following gonorrhea, the duration of infection may influence the magnitude of several immunologic responses which, in turn, may play a role in acquired immunity. A longer duration of $N$. gonorrhoeae infection has been postulated to be one reason for greater humoral $(6,22-24)$ and celluar (25) immune responses in certain individuals. Reinfection in men may therefore be related to the fact that the short duration of infection before treatment may not give ample time for the degree of resistance to reinfection observed in the chimpanzees to develop.

A second major difference between our chimpanzee system and naturally acquired disease is that the chimpanzees were infected with a single $N$. gonorrhoeae isolate. The strain specificity of the immunity we describe is unknown, and it could vary from the meningococcal system where immunity is group specific (26, 27) and involves relatively few groups to the pneumococcal system where immunity is type specific and involves a relatively large number of types (28). Repeated episodes of gonococcal urethritis may therefore be related to exposure to different strains which do not share cross-immunity with strains encountered in prior infections.

The third major difference may be the numbers of gonococci transmitted in man as opposed to the numbers transmitted in our experiments with chimpanzees. The acquired immunity to gonococcal urethritis in our experiments, although statistically significant and of great magnitude relative to numbers of gonococci, could be overcome in over half of the cases with a very large gonococcal challenge. The MIDs on rechallenge for those animals reinfected ranged from $4.6 \times 10^{6}$ to $5.1 \times 10^{8} \mathrm{CFU}$, with a geometric mean of $2.8 \times 10^{7}$ 
CFU. A major unknown is the number of gonococci transmitted in the sexual transmission of the disease in man. These numbers of gonococci may exceed those encountered in infections transmitted by nasopharyngeal secretion droplets, such as streptococcal, pneumococcal, and meningococcal diseases. If the numbers of gonococci sexually transmitted are in the range of $10^{8}$ or greater, the immune system could be overwhelmed and reinfection could possibly occur despite a high degree of immunity. Data on the numbers of gonococci sexually transmitted are not available.

Previous investigations in man have shown that colony types 1 and 2 of a single isolate (F62) are capable of inducing gonococcal urethritis and therefore contrast with types 3 and 4 which, after 69 in vitro passes, are unable to produce gonococcal infection (5, $11,12)$. Virulence therefore correlates with colony type. More recent investigations have shown that colony types 1 and 2 have extracellular structures known as pili or fimbriae which are lacking on the avirulent types 3 and $4(13,14)$. This study indicates that gonococcal virulence may be more than a factor of colony type and pili in that inocula of up to $10^{10} \mathrm{CFU}$ of type 1 colonies from two isolates (isolates $\mathrm{C}$ and $\mathrm{T}$ ) did not induce urethritis in the same chimpanzees later infected with type 1 colonies of another isolate (isolate M). Pili were present on the type 1 and 2 colonies of all three isolates. Pili were not present on the colony type 5 of isolate $\mathrm{K}_{3} 43562$. Type 5 (15) is a relatively uncommon gonococcal colony type whose virulence for mucosal surfaces has not been previously evaluated. This study demonstrates that $10^{10} \mathrm{CFUs}$ of $\mathrm{K}_{2} 43562$ colony type 5 were avirulent in the chimpanzee.

Our current investigations did not detect significant differences between urethral infections in chimpanzees with gonococcal urethritis $2 \mathrm{yr}$ previously and chimpanzees not previously exposed to $N$. gonorrhoeac. In those instances where MRDs were determined with $\mathrm{M}$ isolate, the geometric mean MRD for those animals previously infected was $1.5 \times 10^{3} \mathrm{CFU}$, and the MRD for a single animal not previously infected was $1.4 \times$ $10^{3} \mathrm{CFU}$. The mean duration of infection with $\mathrm{M}$ isolate in five chimpanzees previously infected was 28.2 days, and the infection duration in five chimpanzees not previously infected was 30.0 days. This failure to demonstrate immunity 2 yr after gonococcal urethritis contrasts sharply with the immunity to $\mathrm{M}$ isolate demonstrated in this study and may be secondary to one or more factors. The rechallenge experiments with $\mathrm{M}$ isolate detailed in this study were initiated within a week of the second negative culture which confirmed spontaneous eradication of the initial infection. The rechallenges were completed within a 2-mo interval, and the duration of the immunity beyond this time was not tested and is unknown. Possibly there is a decline in immunity after a 2-yr interval. Another possibly important variable is that different gonococcal isolates were used in the infections $2 \mathrm{yr}$ previously, and these were not available for rechallenge experiments. If immunity to $N$. gonorrhoeae is strain specific, immunity to the previous inocula could still be present but undetected because of limited or no cross-immunity to $\mathrm{M}$ isolate. Our data does not indicate which of these two mechanisms (or other mechanisms) accounts for the ease of reinfection.

Our main objective was to answer the question of whether immunity to gonococcal reinfection is acquired. This was done because the presence of acquired immunity in other diseases suggested that acquired immunity via vaccine might be feasible. Conversely, the vaccine approach has not been as successful in diseases in which immunity to reinfection is nonexistent or of limited value. Our data on acquired immunity suggest that the vaccine approach may be of value in the control of the gonorrhea epidemic. This approach may be the only answer, since the disease is increasing (29) despite effective means for diagnosis and treatment.

\section{ACKNOWLEDGMENTS}

The authors acknowledge the assistance of A. Armstead, R. Cook, and Dr. U. S. G. Kuhn.

\section{REFERENCES}

1. Kearns, D. H., G. B. Seibert, R. O'Reilly, L. Lee, and L. Logan. 1973. Paradox of the immune response to uncomplicated gonococcal urethritis. N. Engl. J. Mcd. 289: $1170-1174$.

2. Holmes, K. K., D. W. Johnson, and H. J. Trostle. 1970. Estimate of the risk of men acquiring gonorrhea by sexual contact with infected females. Am. J. Epidcmiol. 91: 170-174.

3. Balfour, F. 1767. De Gonorrhoea Virulenta. Balfour, Auld and Smellie, Edinburgh. 27 pp.

4. Mahoney, J. F., C. J. Van Slyke, J. C. Cutler, and H. L. Blum. 1946. Experimental gonococci urethritis in human volunteers. Am. J. Syph. Gonor. Vener. Dis. 30: 1-39.

5. Kellogg, D. S., I. R. Cohen, L. C. Norins, A. L. Schroeter, and G. Reising. 1968. Neisseria gonorrhocae. II. Colonial variation and pathogenicity during 35 months in vitro. J. Bacteriol. 96: 596-605.

6. Cohen, I. R., D. S. Kellogg, and L. C. Norins. 1969. Serum antibody response in experimental human gonorrhea. Br. J. Vener. Dis. 45: 325-327.

7. Mahoney, J. F., C. J. Van Slyke, R. R. Wolcott, J. D. Thayer, and A. Nimelman. 1942. Culture studies in chronic gonorrhea of women. Am. J. Syph. Gonor. Vener. Dis. 26: 38-47.

8. Lucas, C. T., F. W. Chandler, J. E. Martin, and J. D. Schmale. 1971. Transfer of gonococcal urethritis from man to chimpanzee. JAMA J. Am. Med. Assoc. 216: $1612-1614$. 
9. Brown, W. J., C. T. Lucas, and U. S. G. Kuhn. 1972 Gonorrhea in the chimpanzee. Infection with laboratorypassed gonococci and by natural transmission. $B r . J$. Vener. Dis. 48 : 177-178.

10. Brown, W. J., and C. T. Lucas. 1973. Gonorrhea in the chimpanzee. Serological testing. Br. J. Vener. Dis. 49 : 441-445.

11. Kellogg, D. S., W. L. Peacock, W. E. Deacon, L Brown, and C. I. Pirkle. 1963. Neisseria gonorrhoeae. I. Virulence genitically linked to colonal variation. J. Bacteriol. 85 : 1274-1279.

12. Kellogg, D. S., and J. D. Thayer. 1969. Virulence of gonococci. Annu. Rev. Med. 20: 323-328.

13. Swanson, J., S. J. Kraus, and E. C. Gotschlich. 1971 Studies on gonococcus infection I. Pili and zones of adhesion: their relation to gonococcal growth patterns. J. Exp. Med. 134 : 886-906.

14. Jephcott, A. E., A. Peyn, and A. Birch-Anderson. 1971. Brief report: Neisseria gonorrhoeae. III. Demonstration of presumed appendages to cells from different colony types. Acta Pathol. Microbiol. Scand. Sect. B Microbiol. Immunol. 79: 437-439.

15. Jephcott, A. E., and A. Reyn. 1971. Neisseria gonorrhoeae. Colony variation I. Acta Pathol. Microbiol. Scand. Sect. B Microbiol. Immunol. 79: 609-614.

16. Thayer, J. D., and J. E. Martin. 1966. Improved medium selective for cultivation of $N$. gonorrhoeae and N. meningitidis. Public Health Rep. 81: 559-562.

17. Kovalchik, M. T., and S. J. Kraus. 1972. Neisseria gonorrhoeae: colonial morphology of rectal isolates. Appl. Microbiol. 23 : 986-989.

18. Riddell, R. H., and A. C. Buck. 1970. Trimethoprim as an additional selective agent in media for the isolation of N. gonorrhoeae. J. Clin. Pathol. (Lond.). 23 $481-483$.
19. Volk, J., and S. J. Kraus. 1974. Asymptomatic meningococcal urethritis. Br. J. Vener. Dis. 49:511-512.

20. Welander, E. 1884. Nagra undersoknigar om gonorrhens patogena mikrober, Nordiskt. Med. Arh. 16: 1-41.

21. Volk, J., and S. J. Kraus. 1974. A venereal disease as prevalent as epidemic gonorrhea-nongonococcal urethritis. Arch. Intern. Med. 134: 511-514.

22. Hess, E. V., D. K. Hunter, and M. Ziff. 1965. Gonococcal antibodies in acute arthritis. JAMA J. Am. Med. Assoc. 191 : 531-534.

23. Magnusson, B., and J. Kjellander. 1965. Gonococcal complement-fixation test in complicated and uncomplicated gonorrhoeae. Br. J. Vener. Dis. 41: 127-131.

24. Buchanan, T. M., J. Swanson, K. K. Holmes, S. J. Kraus, and E. C. Gotschlich. 1973. Quantitative determination of antibody to gonococcal pili. J. Clin. Invest. 52: 2896-2909.

25. Kraus, S. J., G. H. Perkins, and R. C. Geller. 1970. Lymphocyte transformation in repeated gonococcal urethritis. Infect. Immun. 2: 655-658.

26. Goldschneider, I., E. C. Gotschlich, and M. S. Artenstein. 1969. Human immunity to the meningococcus. II. Development of natural immunity. J. Exp. Med. 129: 1327-1348.

27. Gotschlich, E. C., I. Goldschneider, and M. S. Artenstein. 1969. Human immunity to the meningococcus. V. The effect of immunization with meningococcal group C polysaccharide on the carrier state. J. Exp. Med. 82: 1385-1395.

28. MacLeod, C. M., R. G. Hodges, M. Heidelberger, and W. G. Bernard. 1945. Prevention of pneumococcal pneumonia by immunization with specific capsular polysaccharides. J. Exp. Med. 82: 445-465.

29. Center for Disease Control. 1974. Morbility and mortality. Weekly Report. 23: 77. 